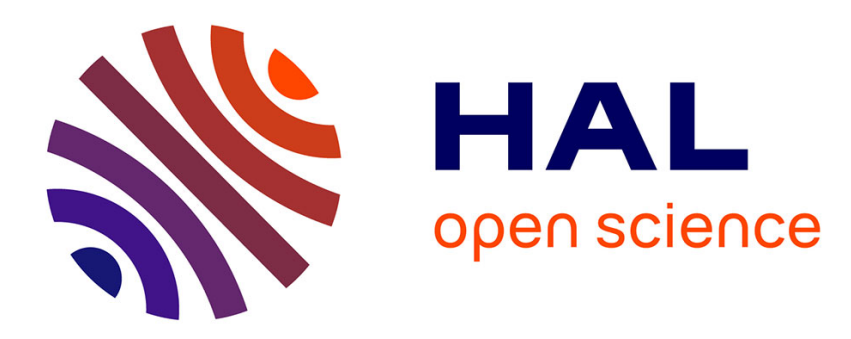

\title{
Morphogenesis of Growing Soft Tissues
}

Julien Dervaux, Martine Ben Amar

\section{To cite this version:}

Julien Dervaux, Martine Ben Amar. Morphogenesis of Growing Soft Tissues. Physical Review Letters, 2008, 101 (6), 10.1103/PhysRevLett.101.068101 . hal-02326607

\section{HAL Id: hal-02326607 https://hal.science/hal-02326607}

Submitted on 22 Oct 2019

HAL is a multi-disciplinary open access archive for the deposit and dissemination of scientific research documents, whether they are published or not. The documents may come from teaching and research institutions in France or abroad, or from public or private research centers.
L'archive ouverte pluridisciplinaire HAL, est destinée au dépôt et à la diffusion de documents scientifiques de niveau recherche, publiés ou non, émanant des établissements d'enseignement et de recherche français ou étrangers, des laboratoires publics ou privés. 


\title{
Morphogenesis of Growing Soft Tissues
}

\author{
Julien Dervaux and Martine Ben Amar \\ Laboratoire de Physique Statistique, Ecole Normale Supérieure, 24 rue Lhomond, 75231 Paris Cedex 05, France
}

(Received 12 December 2007; published 5 August 2008)

\begin{abstract}
Recently, much attention has been given to a noteworthy property of some soft tissues: their ability to grow. Many attempts have been made to model this behavior in biology, chemistry, and physics. Using the theory of finite elasticity, Rodriguez has postulated a multiplicative decomposition of the geometric deformation gradient into a growth-induced part and an elastic one needed to ensure compatibility of the body. In order to fully explore the consequences of this hypothesis, the equations describing thin elastic objects under finite growth are derived. Under appropriate scaling assumptions for the growth rates, the proposed model is of the Föppl-von Kármán type. As an illustration, the circumferential growth of a free hyperelastic disk is studied.
\end{abstract}

DOI: 10.1103/PhysRevLett.101.068101

PACS numbers: 87.19.1x, 68.55.at, 87.19.R-

Biological tissues are conventionally classified into two categories: hard tissues (e.g., bones or teeth) and soft tissues (e.g., muscles, arteries, tendons, or skin), depending on their mechanical properties. Soft tissues, which typically exhibit anisotropic, nonlinear, inhomogeneous behaviors, are often subject to large stresses and strains. The theory of finite elasticity therefore forms an appropriate framework to describe their properties [1-3], in the absence of viscoelastic effects. Along these lines, much work has been done to establish constitutive relationships for specific biological materials such as the skin, blood vessels, lung, brain, liver, and kidney $[3,4]$, although computing stresses and strains under applied external loads remains a difficult task.

Observation of biological tissues has revealed the existence of internal stresses, even in the absence of external loads. These residual stresses are induced by growth [2] and affect the geometrical properties of tissues. Soft tissues may undergo volumetric growth $[5,6]$ depending on space, orientation, and the state of stress within the body. Growth is a complex process involving biochemical and physical reactions at many different length and time scales that occur through cell division, cell enlargement, secretion of extracellular matrix, or accretion at surfaces. The removal of mass is referred to as atrophy and occurs through cell death, cell shrinkage, or resorption. Because of completely different time scales between relaxation via viscoelastic effects and the growth process itself which is assumed very slow, the total deformation of the body is only due to both change of mass and elastic deformations [7-12].

Before (respectively, after) the deformation, the body is in the reference (respectively, current) configuration, and the place of each material point is denoted by $\mathbf{X}$ (respectively, $\mathbf{x}$ ). We define the geometric deformation tensor by $\mathbf{F}=\partial \mathbf{x} / \partial \mathbf{X}$ to describe locally the overall deformation process. In order to model the growth process, we follow Rodriguez, Hoger, and McCulloch [13] in making the following three assumptions: (i) There exists a zero-stress reference state; (ii) the geometric deformation gradient $\mathbf{F}$ admits a multiplicative decomposition of the form $\mathbf{F}=$ AG, where $\mathbf{G}$ is a growth tensor describing the change in mass and $\mathbf{A}$ an elastic tensor characterizing the reorganization of the body needed to ensure compatibility (no overlap) and integrity (no cavitation) of the body; (iii) the response function of the material depends only on the elastic part of the total deformation. Despite its simplicity, the Rodriguez theory is yet to be investigated, because of the complexity of finite elasticity although inhomogeneous and anisotropic growth has been studied in details in some simple geometry [14,15]. More sophisticated and time-dependent approaches also based on the Rodriguez hypothesis have been achieved for spheroids and cylinders $[16,17]$. Here we focus on growing thin samples subject to slow growth-induced finite displacements, and we assume that the sample has time to relax to its equilibrium shape. This reduction of dimensionality allows us to derive the equilibrium equations whatever the constitutive laws of the tissues.

Under appropriate scaling assumptions, the resulting equations are found to be an extension of the well-known Föppl-von Kármán (FvK) model, a powerful theory for buckling instabilities, that are widely diffused in nature (Fig. 2), but which is also able to explain complex postbuckling phenomena such as crumpling. Experimentally, it has been shown that growth may affect curvature in various systems. In growing gels, both homogeneous growth under constraints [18] and free inhomogeneous growth [19] have been investigated. Thermal expansion [Fig. 1(a)], as well as desiccation, can also bend an elastic body and cause it to crumple as seen in dead leaves [Fig. 1(c)]. In living tissues, viruses such as the cotton leaf crumple virus (CLCrV) modify the growth process, and infected plants exhibit curled or crumpled leaves [Fig. 1(d)], but buckling can also occur during normal development. Some mushrooms' or algae's caps [Fig. 1(b)] may undergo symmetry breaking and adopt an oscillatory or cup shape. At the cellular level, a new milestone was reached with the discovery of the CINCINNATA gene whose local expression affects growth 
(a)

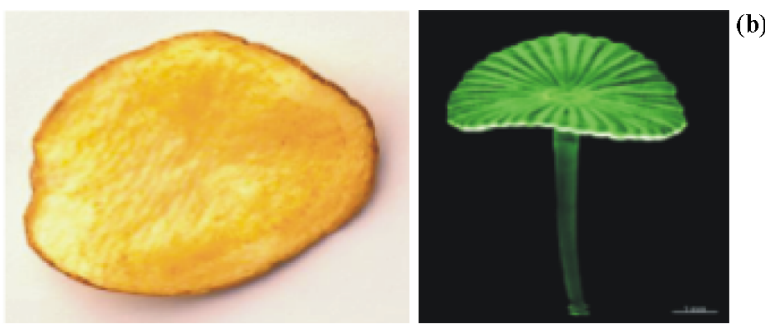

(c)

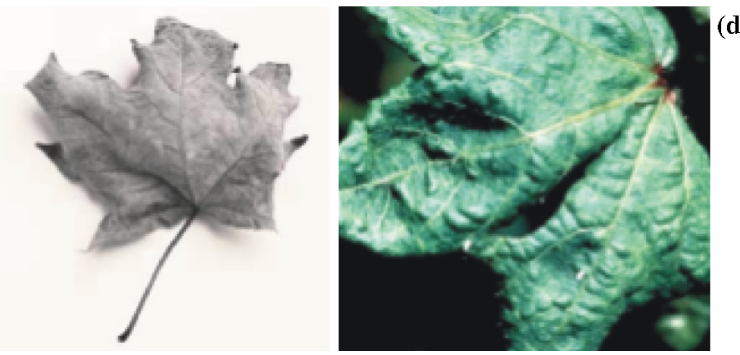

FIG. 1 (color online). A few examples of buckling in nature. (a) A potato chip adopts a saddle shape during frying. (b) Acetabularia schenckii: a green algae. (c) A dead leaf. (d) A leaf infected by the CLCrV, known to affect growth and induce curling and the appearance of blisters at the surface of the leaf.

and curvatures of the Antirrhinum (snapdragon) leaf [20]. Complementary to the inhomogeneity of growth, anisotropy has been shown to be crucial in the generation of shape. Indeed "a key aspect of shape — petal asymmetry in the petal lobe of Antirrhinum depends on the direction of growth rather than regional differences in growth rate" [21]. To investigate the effects of anisotropy, for which our formalism is well suited, we study the problem of a free elastic disk subject to homogeneous anisotropic growth.

The model. - Since biological soft tissues have a high volume fraction of water, they are elastically incompressible; in our notation $\operatorname{det} \mathbf{A}=1$. Furthermore, we assume isotropy of the material for simplicity, and we define a strain energy function: $\mathcal{W}=\sum_{r, s=0}^{\infty} c_{r s}\left(I_{1}-3\right)^{r} \times$ $\left(I_{2}-3\right)^{s}, I_{1}$ and $I_{2}$ being the principal invariants of the tensor $\mathbf{A}^{t} \mathbf{A}$. Any of the common constitutive relationships can be described by a series of this form [22], at least locally. After the deformation process, the sheet, of lateral size $L$ and thickness $H$, is described by the displacement field: $\mathbf{u}=\mathbf{x}-\mathbf{X}$, and we define $\zeta(X, Y)=u_{Z}(X, Y, 0)$ as the displacement of the middle surface that we assume smaller than $L$. When the growth rates $g_{i j}=G_{i j}-\delta_{i j}$ are in the range of or less than $(\zeta / L)$ when one of the indices is 3 and $(\zeta / L)^{2}$ otherwise, the scaling of the induced strains falls inside the domain of validity of the FvK model. Since $H \ll L$, we also apply the membrane assumption that states $\sigma_{X Z}=\sigma_{Y Z}=\sigma_{Z Z}=0$. Then, using this assumption and to leading orders, we find that all materials behave according to the constitutive equation $\boldsymbol{\sigma} \sim(2 Y / 3)(\mathbf{E}-p \mathbf{I}), p$ being the hydrostatic pressure associated with the incompressibility constraint and $\mathbf{E}$ the in-plane Green tensor. $Y$, the instantaneous Young modulus, is equal to: $Y=6\left(c_{01}+c_{10}\right)$. Thus all thin elas- tic samples undergoing small (but finite) deflections follow a generalized Hooke's law whatever the constitutive relationship is, as previously noted in Ref. [23]. The pressure is given by the assumptions $\sigma_{Z Z}=0$, which implies that $p=$ $E_{Z Z}$. Once these results are established, we derive the equilibrium equations using the principle of minimal energy. They can be written in terms of the off-plane displacement and stresses:

$$
\begin{aligned}
D\left(\Delta^{2} \zeta-\Delta \phi\right)-H \partial_{X_{\beta}}\left(\sigma_{\alpha \beta} \partial_{X_{\alpha}} \zeta\right) & =P, \\
\partial_{X_{\beta}} \sigma_{\alpha \beta} & =0,
\end{aligned}
$$

where the Einstein summation convention is used, indices run from 1 to $2, D=Y H^{3} / 9$ is the bending rigidity of the plate, and $\phi$ is a source of mean curvature linked to the growth tensor via $\phi=\operatorname{Div}\left(\mathbf{G G}^{t}\right) \cdot \mathbf{e}_{Z} \cdot \Delta$ and $\Delta^{2}$ mean, respectively, the Laplacian and the bi-Laplacian operator, and $P$ is a possible external loading pressure acting on the plate. At this stage, except from the induced average curvature $\phi$, we recover the FvK equations [24], and we can transform this system (1a) and (1b) with the help of the Airy potential defined by: $\sigma_{\alpha \beta}=\epsilon^{\alpha, \delta} \epsilon^{\beta, \gamma} \partial^{2} \chi / \partial X_{\delta} \partial X_{\gamma}$ ( $\epsilon^{\alpha, \delta}$ being the Levi-Civita tensor). With $\chi$, we derive

$$
\begin{aligned}
D\left(\Delta^{2} \zeta-\Delta \phi\right)-2 H[\chi, \zeta] & =P, \\
\Delta^{2} \chi+E([\zeta, \zeta]-\psi) & =0,
\end{aligned}
$$

where the [., .] operator is defined in Refs. [24,25] and the function $\psi$ appearing in (2) is a source of Gaussian curvature. It is the Gaussian curvature of the distorted surface whose first fundamental form is given by $d x^{2}=$ $G_{\alpha \beta} G_{\alpha \gamma} d X_{\beta} d X_{\gamma}$ [26]. Calculated to leading orders using Brioschi's formula and the Gauss-Bonnet theorem [26], its value in the Cartesian coordinate system is given explicitly in Ref. [25] for an arbitrary nondiagonal growth tensor $G$. The fact that $\psi$ is a Gaussian curvature while $\phi$ is the $z$ component of the divergence of a tensor proves that both quantities are intrinsic quantities associated to the growth tensor and are independent of the choice of coordinate systems as it is the case for all operators in the Föpplvon Kármán equations.

The sets of equilibrium equations (1) and (2) are a generalization of the well-known FvK theory of thin plates, to which they reduce in absence of growth, i.e., $\mathbf{G}=\mathbf{I}$ $(\phi=\psi=0)$. For large deformations $(\zeta \gg H)$, the problem can be simplified. Indeed, the bending term $D\left(\Delta^{2} \zeta-\right.$ $\Delta \phi)$ can be neglected, and a solution that cancels the inplane stresses is a solution of $[\zeta, \zeta]=\psi$, called a MongeAmpère equation. Once this equation is solved, the parameters appearing in this solution can be selected through minimization of the bending energy. For moderate deflections, i.e., $\zeta \sim H$, both bending and stretching terms are of the same order, and the solution of zero energy is a surface with prescribed curvatures, which does not always exist; for example, there is no surface that has positive Gaussian curvature and zero mean curvature. It is known that in- 
homogeneous growth can lead to sophisticated surface geometries [19], so we focus the research on anisotropic growth, which has been much less studied.

The free disk. - Consider a disk, of initial radius $R_{i}$, subject to anisotropic homogeneous growth, with free boundaries and no external loading. Referring to a cylindrical system of coordinates $(R, \Theta, Z)$, the growth tensor is diagonal and homogeneous: $\mathbf{G}=\operatorname{diag}\left(1+g_{1}, 1+g_{2}, 1\right)$ neglecting the thickening of the plate. If $g_{1}$ and $g_{2}$, respectively, the radial and circumferential components of the growth process, are equal, then growth is homogeneous and isotropic, and no residual stress appears: The disk remains flat. The relevant control parameter is $k=g_{2}-$ $g_{1}$. The first case to consider is for $k \ll H^{2} / R_{i}^{2}$ that induces an off-plane displacement $\zeta$ much smaller than $H$ and is thus outside the scope of the present theory. When $k$ is of order $H^{2} / R_{i}^{2}$, which leads to $\zeta \sim H$, all of the contributions are of the same order, and a linear stability analysis is performed. We look for a solution in which the in-plane fields (displacements $U_{R}$ and $U_{\Theta}$ and stresses $\sigma_{R R}, \sigma_{R \Theta}$, and $\sigma_{\Theta \Theta}$ ) are independent of $\Theta$. The off-plane displacement, however, can depend on $\Theta$. Since the disk is free, the boundary conditions imply that there is no tension or torque at the free edge and reads $\sigma_{R R}\left(R_{i}\right)=\sigma_{R \Theta}\left(R_{i}\right)=$ 0 . The only convergent solution of (1b) that fulfills these boundary conditions is $\sigma_{R R}=\sigma_{R \Theta}=0$ leading to $U_{R}(R)=(2 R / 3)\left(g_{2} / 2+g_{1}\right), U_{\Theta}(R)=0$, and a nonzero hoop stress $\sigma_{\Theta \Theta}=(-2 k Y) / 3$. Assuming a solution with discrete axial symmetry: $\zeta(\rho, \Theta)=\xi(\rho) \cos (m \Theta)$ (with $\rho=R / R_{i}$ ), Eq. (1a) reduces to

$\rho^{4} \xi^{(4)}+2 \rho^{3} \xi^{(3)}-\left(1+2 m^{2}\right) \rho^{2} \xi^{(2)}$

$+\left(1+2 m^{2}+\alpha \rho^{2}\right) \rho \xi^{(1)}+\left(m^{4}-4 m^{2}-m^{2} \alpha \rho^{2}\right) \xi=0$,

where $\alpha=\left(6 k R^{2}\right) / H^{2}$ is a control parameter and $\xi^{(i)}$ is the $i$ th derivative of $\xi$ with respect to $\rho$. At the free edge ( $\rho=$ 1 ), the zero-torque conditions are not affected by the growth process and are described in Ref. [24]. To avoid singularities at $\rho=0$, we impose $\xi(0)=0$ and $\xi^{\prime}(0)=0$. These boundary conditions, together with Eq. (3), form an eigenvalue problem for the threshold $\alpha$. Using Frobenius's method [27], we find the four eigenfunctions corresponding to each $m$. The most unstable mode, occurring when growth is mainly circumferential $(\alpha>0)$, is characterized by $m=2-$ a saddle shape - with a threshold value of $\alpha=3.08$. An axially symmetric solution, i.e., $m=0$, appears when radial growth dominates $(\alpha<0)$, at the threshold value $\alpha=-7.82$. This simple model explains surprisingly well the changes of cap shape that the algae Acetabularia acetabulum undergoes during its development. Experiments performed in Ref. [28] show that radial growth occurs in the earliest stage of the development, which leads to a symmetric conical shape. At a later stage, however, circumferential growth predominates to produce the saddle shape (see Fig. 2).

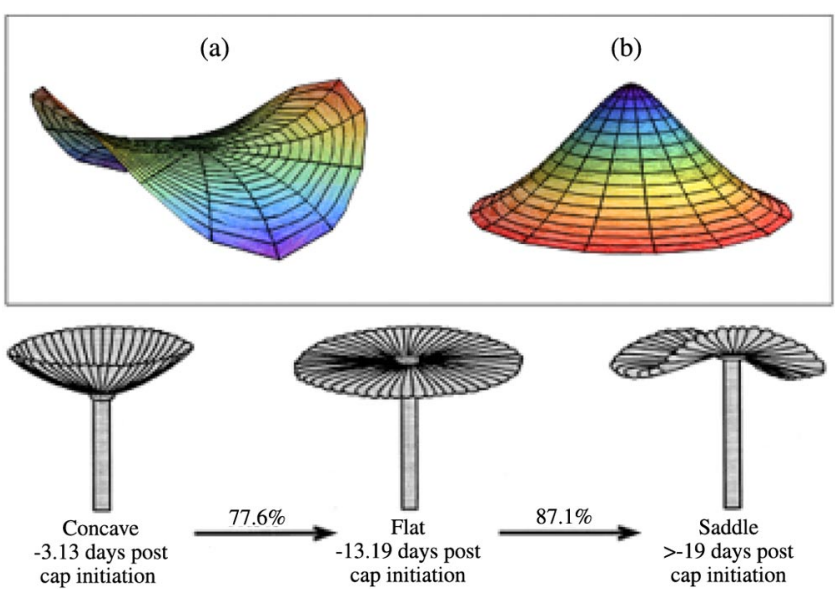

FIG. 2 (color online). Top: The two first destabilized modes. (a) On the left $k>0$, and the disk adopts a saddle shape, with $m=2$, at the threshold $\alpha=3.08$. (b) On the right $k<0$, and the disk adopts an axially symmetric shape characterized by $m=0$, at the threshold $\alpha=-7.82$. Bottom: Shape changes in the Acetabularia algae; the figures indicate the fraction of algae that undergo the shape transition from an initial population of 85 plants. Picture drawn from Ref. [28].

We now consider large deformations: $1 \gg k \gg H^{2} / R_{i}^{2}$, for which $H \ll \zeta$. Since the stretching contribution is much bigger than the bending energy, we first solve the Monge-Ampère equation $[\zeta, \zeta]=\psi$ in which $\psi$ is given by $\psi=k \delta(\rho) / \rho$ in our case. The general solution is a cone that has zero Gaussian curvature except at the tip of the cone, where the effect of bending becomes important and which would require a more precise treatment $[29,30]$. We focus only on the outer solution. The equation of the cone is simply $\zeta(\rho, \Theta)=\rho g(\Theta)$. Using this expression, the condition that the Airy potential vanishes everywhere [so (2a) and (2b) are satisfied] gives for the in-plane displacement field:

$$
\begin{aligned}
U_{\rho} & =-\frac{\rho}{2} g(\Theta)^{2}, \\
U_{\Theta} & =\frac{\rho}{2} \int^{\Theta}\left[g(\Theta)^{2}-g^{\prime}(\Theta)^{2}+2 k\right] d \Theta .
\end{aligned}
$$

Periodicity in the orthoradial displacement implies that $U_{\Theta}(\rho, 0)=U_{\Theta}(\rho, 2 \pi)$. Let $g(\Theta)$ be represented by its Fourier series: $g(\Theta)=\sum_{n=0}^{\infty}\left(a_{n} e^{i n \Theta}+a_{n}^{\star} e^{-i n \Theta}\right)$. The periodicity condition leads to $2 \sum_{n=1}^{\infty}\left[a_{n} a_{n}^{\star}\left(n^{2}-1\right)\right]=$ $\left(a_{0}+a_{0}^{\star}\right)^{2}+2 k$. A cone of revolution (for which $a_{0}$ is the only nonzero coefficient of the Fourier series) can satisfy the periodicity condition only if $k<0$, that is, when radial growth dominates. Infinitely many solutions satisfy the periodicity condition, but the selected shape must have minimal bending energy [29-31]. The bending contribution reads $\mathcal{E}_{b} \propto\left\{2 \sum_{n=1}^{\infty}\left[a_{n} a_{n}^{\star}\left(n^{2}-1\right)^{2}\right]+\left(a_{0}+\right.\right.$ $\left.a_{0}^{\star}\right)^{2}$ \}. For negative $k$ values, the solution of minimal outer bending energy is given by $\zeta(R, \Theta)=R \sqrt{2|k|}$ and for 


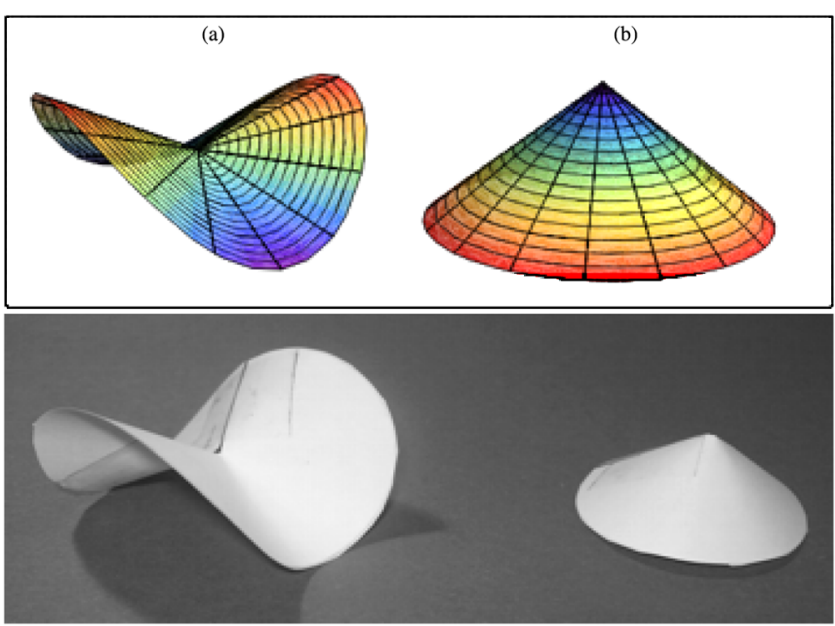

FIG. 3 (color online). Top: The two modes of minimal energy, far from the tip. (a) $k>0$, and the shape is a cone with two oscillations. (b) $k<0$, and the cone of revolution is a solution. Bottom: The resultant shapes built out of paper $(k=0.25)$ are in agreement with the prediction.

positive values by $\zeta(R, \Theta)=R \sqrt{4 k / 3} \cos 2 \Theta$. For large deformations, those predictions can be easily checked by constructing a cone from a disk of paper in which a sector defined by two radii is withdrawn and then either replaced by a bigger one or just glued to close it (see Fig. 3). This simple demonstration illustrates the fact that singularities can arise from growth as observed in dead leaves or in the leaves infected by the CLCrV.

Conclusion.-Using the formalism introduced by Rodriguez, Hoger, and McCulloch, we have developed a theory describing the behavior of thin elastic bodies subject to growth. By explicating the sheet's small thickness, we showed that all materials behave according to a generalized Hooke's law and the equilibrium equations generalize the FvK equations with growth. This extension describes a broad range of physical phenomena involving mass reorganization, from biological growth to thermal dilatation, as well as desiccation. Once observed in experiments, shape instabilities with a well-defined wavelength may give relevant information on the growth process itself. The treatment presented in this Letter also includes growth anisotropic effects. We have shown that anisotropic growth induces rich structures such as curling and crumpling.

We thank A. Boudaoud, P. Ciarletta, and E. Sharon for many enlightening discussions.

[1] Y.C. Fung, Biomechanics: Motion, Flow, Stress, and Growth (Springer, New York, 1990).

[2] R. Skalak, A. Tozeren, R. P. Zarda, and S. Chien, Biophys. J. 13, 245 (1973).
[3] Y.C. Fung, Biomechanics: Material Properties of Living Tissues (Springer, New York, 1993).

[4] K. Miller, Med. Sci. Monitor 6, 158 (2000).

[5] J. D. Humphrey, Proc. R. Soc. A 459, 3 (2003).

[6] S. C. Cowin, Annu. Rev. Biomed. Eng. 6, 77 (2004).

[7] F. H. Hsu, J. Biomech. 1, 303 (1968).

[8] S. C. Cowin and D. H. Hegedus, J. Elast. 6, 313 (1976).

[9] R. Skalak, in Proceedings of the IUTAM Symposium on Finite Elasticity, edited by D. E. Carlson and R. T. Shield (Martinus Nijhoff, The Hague, 1981), p. 347.

[10] V. M. Entov, Mech. Solids 18, 199 (1983).

[11] A. D. Drozdov and H. Khanina, Math. Comput. Model. 25, 11 (1997).

[12] A. A. Stein, J. Appl. Math. Mech. 59, 139 (1995).

[13] E. K. Rodriguez, A. Hoger, and A. McCulloch, J. Biomech. 27, 455 (1994).

[14] M. Ben Amar and A. Goriely, J. Mech. Phys. Solids 53, 2284 (2005).

[15] A. Goriely and M. Ben Amar, Phys. Rev. Lett. 94, 198103 (2005).

[16] D. Ambrosi and F. Mollica, Int. J. Eng. Sci. 40, 1297 (2002).

[17] D. Ambrosi and F. Guana, Math. Mech. Solids 12, 319 (2007).

[18] T. Mora and A. Boudaoud, Eur. Phys. J. E 20, 119 (2006).

[19] D. Drasdo, Phys. Rev. Lett. 84, 4244 (2000); E. Sharon, B. Roman, M. Marder, G. S. Shin, and H. L. Swinney, Nature (London) 419, 579 (2002); Y. Klein, E. Efrati, and E. Sharon, Science 315, 1116 (2007).

[20] U. Nath, B.C. W. Crawford, R. Carpenter, and E. Coen, Science 299, 1404 (2003).

[21] E. Coen, A. G. Rolland-Lagan, M. Matthews, J. A. Bangham, and P. Prusinkiewicz, Proc. Natl. Acad. Sci. U.S.A. 101, 4728 (2004).

[22] R.W. Ogden, Non-linear Elastic Deformations (Dover, New York, 1997).

[23] H. A. Erbay, Int. J. Eng. Sci. 35, 151 (1997).

[24] L. Landau and E. Lifchitz, Théorie de l'elasticité (Mir, Moscow, 1990).

[25] $[f, g]=1 / 2\left[\left(\partial_{x^{2}}^{2} f\right)\left(\partial_{y^{2}}^{2} g\right)+\left(\partial_{y^{2}}^{2} f\right)\left(\partial_{x^{2}}^{2} g\right)-2\left(\partial_{x y}^{2} f\right)\left(\partial_{x y}^{2} g\right)\right]$, where the subscript means partial derivative with respect to Cartesian coordinates $(x, y)$. In these coordinates, the average induced curvature is $\phi=\partial_{x}\left(g_{31}+g_{13}\right)+\partial_{y}\left(g_{32}+\right.$ $\left.g_{23}\right)$, and the induced Gaussian curvature is $\partial_{x^{2}}\left(g_{22}+\right.$ $\left.g_{32}^{2} / 2\right)+\partial_{y^{2}}\left(g_{11}+g_{31}^{2} / 2\right)-\partial_{x y}\left(g_{12}+g_{21}+g_{31}^{2}\right)$.

[26] A. Gray, Modern Differential Geometry of Curves and Surfaces (CRC Press, Boca Raton, FL, 1993).

[27] E. L. Ince, Ordinary Differential Equations (Dover, New York, 1956).

[28] K. A. Serikawa and D. F. Mandoli, Planta 207, 96 (1998).

[29] M. Ben Amar and Y. Pomeau, Proc. R. Soc. A 453, 729 (1997).

[30] E. Cerda and L. Mahadevan, Phys. Rev. Lett. 80, 2358 (1998).

[31] E. Cerda, S. Chaieb, F. Melo, and L. Mahadevan, Nature (London) 401, 46 (1999). 